\title{
A New Pressure Regularity Criterion of the Three-Dimensional Micropolar Fluid Equations
}

\author{
Junbai Ren \\ School of Statistics, Jiangxi University of Finance and Economics, Nanchang 330013, China \\ Correspondence should be addressed to Junbai Ren; junbai_ren@jxufe.edu.cn
}

Received 30 August 2013; Accepted 29 October 2013

Academic Editor: Suh-Yuh Yang

Copyright (C) 2013 Junbai Ren. This is an open access article distributed under the Creative Commons Attribution License, which permits unrestricted use, distribution, and reproduction in any medium, provided the original work is properly cited.

This paper concerns the regularity criterion of the weak solutions to the three-dimensional (3D) micropolar fluid equations in terms of the pressure. It is proved that if one of the partial derivatives of pressure satisfies $\partial_{3} \pi \in L^{p}\left(0, T ; L^{q}\left(\mathbf{R}^{3}\right)\right)$ with $2 / p+3 / q \leq 2,3<$ $q<\infty, 1<p<\infty$, then the weak solution of the micropolar fluid equations becomes regular on $(0, T]$.

\section{Introduction}

In the past ten years, the mathematical models of fluid dynamics attract more and more attention. As a classic fluid dynamical model, Naiver-Stokes equations [1] are proved as an accurate model in many practical situations, which presume that the derivatives of the components of the velocity are small. However, for certain anisotropic fluids, for example, liquid crystals, which are made up of dumbbell molecules, and some polymeric fluids or fluids containing certain additives in narrow films [2], the constructive relations do not satisfy Stoke Law. In 1960s, Eringen [3] introduced viscous incompressible micropolar fluid flows, a nonNewtonian fluid model with asymmetric stress tensor. From the viewpoint of mathematics, micropolar fluid model is coupled with the incompressible Navier-Stokes equations, microrotational effects, and microrotational inertia. The threedimensional (3D) viscous incompressible micropolar fluid equations are written as

$$
\begin{gathered}
\partial_{t} u-(\nu+\kappa) \Delta u-2 \kappa \nabla \times w+\nabla \pi+u \cdot \nabla u=0, \\
\partial_{t} w-\gamma \Delta w-(\alpha+\beta) \nabla \nabla \cdot w+4 \kappa w-2 \kappa \nabla \times u+u \cdot \nabla w=0, \\
\nabla \cdot u=0,
\end{gathered}
$$

associated with the initial prescribed data

$$
\left.u(x, t)\right|_{t=0}=u_{0},\left.\quad w(x, t)\right|_{t=0}=w_{0} .
$$

Here $u=\left(u_{1}, u_{2}, u_{3}\right), \pi$, and $w=\left(w_{1}, w_{2}, w_{3}\right)$ stand for the divergence-free velocity vector field, the scalar pressure field, and nondivergence free microrotation vector field, respectively. $\nu>0$ is the Newtonian kinetic viscosity, $\kappa>0$ is the dynamics microrotation viscosity, and $\alpha, \beta, \gamma>0$ are the angular viscosity.

Due to their importance in mathematics, there is large literature on the well-posedness and large time behaviors for weak solutions of micropolar fluid equations [4-10]. However, the question of global regularity or uniqueness of weak solutions of three-dimensional micropolar fluid equations is still a challenge open problem. Therefore, it is interesting and natural to consider the regularity criteria for weak solutions of micropolar fluid equations by imposing some growth conditions on the velocity or the pressure. As for the velocity regularity criteria, Dong and Chen [11] (see also [12]) obtained that if the velocity fields satisfy one of the following conditions:

(i) $u \in L^{p}\left(0, T ; L^{q, \infty}\left(\mathbf{R}^{3}\right)\right), \quad \frac{2}{p}+\frac{3}{q}=1$,

$3<q \leq \infty$,

(ii) $\nabla u \in L^{p}\left(0, T ; L^{q, \infty}\left(\mathbf{R}^{3}\right)\right), \quad \frac{2}{p}+\frac{3}{q}=2$,

$$
\frac{3}{2}<q \leq \infty,
$$

(iii) $u \in L^{2 /(1-r)}\left(0, T ; \dot{X}^{-r}\left(\mathbf{R}^{3}\right)\right), \quad r \in(0,1]$, 
then the weak solutions of the micropolar fluid equations (1) and (2) are regular. Here the critical spaces $L^{q, \infty}, X^{-r}$ are Lorentz spaces and Multiplier space. The results were further refined by many authors [13-15] to some large critical spaces such as Besov spaces and Triebel-Lizorkin spaces.

On the other hand, as for the pressure regularity criteria of the micropolar fluid equations, Yuan [12] showed that the weak solution becomes regular if the pressure satisfies

$$
\begin{array}{r}
\nabla \pi \in L^{p}\left(0, T ; L^{q, \infty}\left(\mathbf{R}^{3}\right)\right), \quad \text { for } \frac{2}{p}+\frac{3}{q} \leq 3, \\
1<q \leq \infty .
\end{array}
$$

Dong et al. $[16,17]$ improved the regularity criteria by imposing the growth conditions in the critical Besov spaces

$$
\pi \in L^{1}\left(0, T ; B_{\infty, \infty}^{0}\left(\mathbf{R}^{3}\right)\right)
$$

Recently, some interesting logarithmical pressure regularity criteria $[18,19]$ of micropolar fluid equations are studied. In particular, Jia et al. [20] refined this question by imposing the following regularity criterion condition:

$$
\begin{aligned}
\int_{0}^{T} \frac{\left\|\partial_{3} \pi\right\|_{L^{q}}^{p}}{1+\ln \left(e+\|w\|_{L^{4}}\right)} d s<\infty, & \frac{2}{p}+\frac{3}{q}=\frac{7}{4} \\
\frac{12}{7}<q \leq \infty & <.
\end{aligned}
$$

Compared with the results (4) and (6), it is natural to consider whether or not the growth condition of the partial derivative of the pressure $\partial_{3} \pi$ can be released. It should be mentioned that the optimal result is that the regularity for weak solutions of three-dimensional micropolar fluid equations is valid if

$$
\begin{aligned}
\partial_{3} \pi \in L^{p}\left(0, T ; L^{q}\left(\mathbf{R}^{3}\right)\right), & \frac{2}{p}+\frac{3}{q} \leq 3, \\
& 1<q \leq \infty .
\end{aligned}
$$

This is an open problem on the pressure regularity criterion of micropolar fluid equations. The aim of this paper is to understand this challenge problem. More precisely, we will show the regularity of weak solutions to three-dimensional micropolar fluid equations if one of the partial derivatives of the pressure, say, $\partial_{3} \pi$, satisfies

$$
\begin{array}{r}
\partial_{3} \pi \in L^{p}\left(0, T ; L^{q}\left(\mathbf{R}^{3}\right)\right), \quad \frac{2}{p}+\frac{3}{q} \leq 2, \\
3<q<\infty, 1<p<\infty .
\end{array}
$$

One may also refer to some important results on the regularity criteria of some mathematical models in fluid dynamics. For example, Cao and Titi [21], Chen and Zhang [22], Fan et al. [23], and Zhou [24] investigated the regularity criteria for the classic Navier-Stokes equations, Chen et al. [25], He and Xin [26], and Jia and Zhou [27, 28] for MHD equations, Dong et al. $[29,30]$ for quasigeostrophic equation, and so on.

\section{Preliminaries and Main Results}

In this paper, we use the following usual notations. $C$ is the abstract constant which may change from line to line. $L^{p}\left(\mathbf{R}^{3}\right)(1 \leq p \leq \infty)$ is the scalar or vector Lebesgue space of all $L^{p}$ integral functions associated with the norm

$$
\|f\|_{L^{p}}= \begin{cases}\left(\int_{\mathbf{R}^{3}}|f(x)|_{L^{p}} d x\right)^{1 / p}, & 1 \leq p<\infty, \\ \operatorname{ess} \sup _{x \in \mathbf{R}^{3}}|f(x)|, & p=\infty .\end{cases}
$$

The following anisotropic Sobolev inequality is due to $\mathrm{Cao}$ and $\mathrm{Wu}[31]$.

Lemma 1 (Cao and $W u[31])$. Suppose $f \in H^{1}\left(\mathbf{R}^{3}\right), \partial_{1} f, \partial_{2} f \in$ $L^{\lambda}\left(\mathbf{R}^{3}\right)$, and $\partial_{3} f \in L^{\mu}\left(\mathbf{R}^{3}\right)$ and for three constants $1 \leq \mu, \lambda, \gamma<$ $\infty$ satisfy

$$
1+\frac{3}{\gamma}=\frac{1}{\mu}+\frac{2}{\lambda},
$$

then there exists a constant $C=C(\mu, \lambda)$ such that the following anisotropic Sobolev inequality:

$$
\|f\|_{L^{\gamma}} \leq C\left\|\partial_{1} f\right\|_{L^{\lambda}}^{1 / 3}\left\|\partial_{2} f\right\|_{L^{\lambda}}^{1 / 3}\left\|\partial_{3} f\right\|_{L^{\mu}}^{1 / 3}
$$

is valid.

To aid the introduction of our main results, let us recall the definition of the weak solutions of the 3D micropolar fluid flows (1) and (2) (see Łukaszewicz [9]).

Definition 2. Let $\left(u_{0}, w_{0}\right) \in L^{2}\left(\mathbf{R}^{3}\right)$ and $\nabla \cdot u_{0}=0$. A pair of vector fields $(u(x, t), w(x, t)$ is termed as a weak solution to the $3 \mathrm{D}$ micropolar fluid equations (1) and $(2)$ on $(0, T)$ if $(u, w)$ satisfies the following properties:

(i) $(u, w) \in L^{\infty}\left(0, T ; L^{2}\left(\mathbf{R}^{3}\right)\right) \cap L^{2}\left(0, T ; H^{1}\left(\mathbf{R}^{3}\right)\right)$;

(ii) $(u, w)$ verifies $(1)$ in the sense of distribution.

The following existence result of micropolar fluid equations is useful for our results.

Lemma 3 (Dong et al. [16]). Assume $3<p<\infty$ and $\left(u_{0}, w_{0}\right) \in L^{p}\left(\mathbf{R}^{3}\right)$ with $\nabla \cdot u_{0}=0$ in the sense of distributions. Then there exist a constant $T>0$ and a unique strong solution $(u, w)$ of the $3 D$ micropolar fluid equations (1) and (2) such that

$$
\begin{gathered}
u \in B C\left([0, T) ; L^{p}\left(\mathbf{R}^{3}\right)\right), \\
t^{1 / 2} \nabla u \in B C\left([0, T) ; L^{p}\left(\mathbf{R}^{3}\right)\right) .
\end{gathered}
$$

Theorem 4. Suppose $T>0$ and $\left(u_{0}, w_{0}\right) \in L^{2}\left(\mathbf{R}^{3}\right) \cap L^{4}\left(\mathbf{R}^{3}\right)$ and $\nabla \cdot u_{0}$ in the sense of distributions and assume $(u, w)$ is a weak solution of the 3D micropolar fluid equations (1) and (2) on $(0, T]$. If one of the partial derivatives of the pressure, say, $\partial_{3} \pi$, satisfies

$$
\begin{array}{r}
\partial_{3} \pi \in L^{p}\left(0, T ; L^{q}\left(\mathbf{R}^{3}\right)\right), \quad \frac{2}{p}+\frac{3}{q} \leq 2, \\
3<q<\infty, 1<p<\infty,
\end{array}
$$

then $(u, w)$ is regular on $[0, T]$. 


\section{Proof of Theorem 4}

We first establish some fundamental estimates between the pressure and the velocity of the micropolar fluid equations (1) and (2). Taking the operator div to both sides of the first equation of (1) and noting the fact of the divergence-free velocity, one shows that

$$
-\Delta \pi=\sum_{i, j=1}^{3} \frac{\partial^{2}}{\partial x_{i} \partial x_{j}}\left(u_{i} u_{j}\right),
$$

together with Calderon-Zygmund inequality, implies that for any $1<p<\infty$

$$
\begin{aligned}
\|\pi\|_{L^{p}} & =\left\|(-\Delta)^{-1} \sum_{i, j=1}^{3} \frac{\partial^{2}}{\partial x_{i} \partial x_{j}}\left(u_{i} u_{j}\right)\right\|_{L^{p}} \\
& \leq C\|u\|_{L^{2 p}}^{2} .
\end{aligned}
$$

Similarly, acting the operator $\nabla \operatorname{div}$ on both sides of the second equation of (1), we have

$$
\begin{aligned}
\nabla \pi & =(-\Delta)^{-1} \sum_{i, j=1}^{3} \frac{\partial^{2}}{\partial x_{i} \partial x_{j}}\left(\nabla\left(u_{i} u_{j}\right)\right), \\
\|\nabla \pi\|_{L^{p}} & =\left\|(-\Delta)^{-1} \sum_{i, j=1}^{3} \frac{\partial^{2}}{\partial x_{i} \partial x_{j}}\left(\nabla\left(u_{i} u_{j}\right)\right)\right\|_{L^{p}} \\
& \leq C\||u| \nabla u\|_{L^{p}} .
\end{aligned}
$$

In order to prove the main result, we also need some auxiliary estimates of the weak solutions of the micropolar fluid equations (1). Thanks to the divergence-free velocity fields and application to the integration by parts, we have the following estimates:

$$
\int_{\mathbf{R}^{3}}(u \cdot \nabla u) \cdot u|u|^{2} d x=0, \quad \int_{\mathbf{R}^{3}}(u \cdot \nabla w) \cdot w|w|^{2} d x=0 .
$$

In particular, by direct computation, we also have

$$
\begin{aligned}
& (\nu+\kappa) \int_{\mathbf{R}^{3}}(-\Delta u) u|u|^{2} d x \\
& =(\nu+\kappa) \int_{\mathbf{R}^{3}}(\nabla u) \cdot\left(\nabla\left(u|u|^{2}\right)\right) d x \\
& \quad=(\nu+\kappa) \int_{\mathbf{R}^{3}}|\nabla u|^{2}|u|^{2} d x+\left.\left.\frac{\nu+\kappa}{2} \int_{\mathbf{R}^{3}}|\nabla| u\right|^{2}\right|^{2} d x, \\
& \gamma \int_{\mathbf{R}^{3}}(-\Delta w) w|w|^{2} d x \\
& \quad=\gamma \int_{\mathbf{R}^{3}}(\nabla w) \cdot\left(\nabla\left(w|w|^{2}\right)\right) d x \\
& \quad=\gamma \int_{\mathbf{R}^{3}}|\nabla w|^{2}|w|^{2} d x+\left.\left.\frac{\gamma}{2} \int_{\mathbf{R}^{3}}|\nabla| w\right|^{2}\right|^{2} d x .
\end{aligned}
$$

We now begin to prove Theorem 4 .
Taking the inner product of the first equation of (1) with $u|u|^{2}$ and the second equation of (1) with $w|w|^{2}$, respectively, it follows that

$$
\begin{aligned}
& \frac{1}{4} \frac{d}{d t} \int_{\mathbf{R}^{3}}|u|^{4} d x+(\nu+\kappa) \\
& \quad \times \int_{\mathbf{R}^{3}}|| u|\nabla u|^{2} d x+\left.\frac{\nu+\kappa}{2} \int_{\mathbf{R}^{3}}|\nabla| u\right|^{2} d x \\
& =2 \kappa \int_{\mathbf{R}^{3}}(\nabla \times w) \cdot u|u|^{2} d x-\int_{\mathbf{R}^{3}} u \cdot \nabla \pi|u|^{2} d x, \\
& \frac{1}{4} \frac{d}{d t} \int_{\mathbf{R}^{3}}|w|^{4} d x+\gamma \int_{\mathbf{R}^{3}}|| w|\nabla w|^{2} d x \\
& +\left.\left.\frac{\gamma}{2} \int_{\mathbf{R}^{3}}|\nabla| w\right|^{2}\right|^{2} d x+\frac{(\alpha+\beta)}{2} \int_{\mathbf{R}^{3}}|\nabla \cdot w|^{2}|w|^{2} d x \\
& \leq 2 \kappa \int_{\mathbf{R}^{3}}(\nabla \times u) \cdot w|w|^{2} d x-4 \kappa \int_{\mathbf{R}^{3}}|w|^{4} d x .
\end{aligned}
$$

Applying Hölder inequality, Young inequality, and integration by parts, one shows that

$$
\begin{aligned}
& 2 \kappa \int_{\mathbf{R}^{3}}(\nabla \times w) \cdot u|u|^{2} d x \\
& \quad+2 \kappa \int_{\mathbf{R}^{3}}(\nabla \times u) \cdot w|w|^{2} d x-4 \kappa \int_{\mathbf{R}^{3}}|w|^{4} d x \\
& \leq 2 \kappa\|w\|_{L^{4}}\||u| \nabla u\|_{L^{2}}\|u\|_{L^{4}} \\
& \quad+2 \kappa\|u\|_{L^{4}}\||w| \nabla w\|_{L^{2}}\|w\|_{L^{4}}-4 \kappa\|w\|_{L^{4}}^{4} \\
& \leq C\left(\|u\|_{L^{4}}^{4}+\|w\|_{L^{4}}^{4}\right)+\frac{\nu+\kappa}{2}\||u| \nabla u\|_{L^{2}}^{2}+\frac{\gamma}{2}\||w| \nabla w\|_{L^{2}}^{2} .
\end{aligned}
$$

Summing up (19) and (20), we have

$$
\begin{aligned}
& \frac{1}{4} \frac{d}{d t}\left(\int_{\mathbf{R}^{3}}|u|^{4} d x+\int_{\mathbf{R}^{3}}|w|^{4} d x\right) \\
& \quad+\frac{\nu+\kappa}{2} \int_{\mathbf{R}^{3}}|| u|\nabla u|^{2} d x+\frac{\gamma}{2} \int_{\mathbf{R}^{3}}|| w|\nabla w|^{2} d x \\
& \leq-\int_{\mathbf{R}^{3}} u \cdot \nabla \pi|u|^{2} d x .
\end{aligned}
$$

Now we estimate the right hand side of (21). Employing Hölder inequality and Young inequality firstly yields

$$
\begin{aligned}
\left.\left|-\int_{\mathbf{R}^{3}} u \cdot \nabla \pi\right| u\right|^{2} d x \mid & \leq C\|\pi\|_{L^{4}}\|u\|_{L^{4}}\left\|\nabla|u|^{2}\right\|_{L^{2}} \\
& \leq C\|\pi\|_{L^{4}}^{2}\|u\|_{L^{4}}^{2}+\frac{\nu+\kappa}{4}\||u| \nabla u\|_{L^{2}}^{2} .
\end{aligned}
$$


Applying interpolation inequality and Lemma 1 together with (14) and (16), it follows that

$$
\begin{aligned}
\|\pi\|_{L^{4}}^{2}\|u\|_{L^{4}}^{2} & \leq C\|\pi\|_{L^{\rho}}^{2(1-\theta)}\|\pi\|_{L^{2}}^{2 \theta}\|u\|_{L^{4}}^{2} \\
& \leq C\|\pi\|_{L^{\rho}}^{2(1-\theta)}\|u\|_{L^{4}}^{4 \theta+2} \\
& \leq C\left\|\partial_{3} \pi\right\|_{L^{q}}^{(2(1-\theta)) / 3}\left\|\nabla_{h} \pi\right\|_{L^{4 / 3}}^{(4(1-\theta)) / 3}\|u\|_{L^{4}}^{4 \theta+2} \\
& \leq C\left\|\partial_{3} \pi\right\|_{L^{q}}^{(2(1-\theta)) / 3}\|\nabla \pi\|_{L^{4 / 3}}^{(4(1-\theta)) / 3}\|u\|_{L^{4}}^{4 \theta+2} \\
& \leq C\left\|\partial_{3} \pi\right\|_{L^{q}}^{(2(1-\theta)) / 3}\||u| \nabla \pi\|_{L^{4 / 3}}^{(4(1-\theta)) / 3}\|u\|_{L^{4}}^{4 \theta+2},
\end{aligned}
$$

where we have used interpolation inequality

$$
\begin{gathered}
\frac{1-\theta}{\rho}+\frac{\theta}{2}=\frac{1}{4} \quad(0 \leq \theta \leq 1), \\
1+\frac{3}{\rho}=\frac{1}{q}+\frac{2}{4 / 3}
\end{gathered}
$$

(let $\gamma=\rho, \mu=q$, and $\lambda=4 / 3$ in Lemma 1 ).

That is to say, we may check $q$ as

$$
q=\frac{4(1-\theta)}{1-4 \theta} \text {. }
$$

With the aid of Hölder inequality and Young inequality, $\|\pi\|_{L^{4}}^{2}\|u\|_{L^{4}}^{2}$ is now further estimated as

$$
\begin{aligned}
\|\pi\|_{L^{4}}^{2}\|u\|_{L^{4}}^{2} \leq & C\left(\left\|\partial_{3} \pi\right\|_{L^{q}}^{(2(1-\theta)) / 3}\|\nabla u\|_{L^{2}}^{(4(1-\theta)) / 3}\right) \\
& \times\left(\|u\|_{L^{4}}^{2}\|u\|_{L^{4}}^{(4+8 \theta) / 3}\right) \\
\leq & C\left(\left\|\partial_{3} \pi\right\|_{L^{q}}^{(2(1-\theta)) /(1+2 \theta)}+\|\nabla u\|_{L^{2}}^{2}\right) \\
& \times\left(\|u\|_{L^{4}}^{4}+\|u\|_{L^{4}}^{(8+16 \theta) / 3}\right) .
\end{aligned}
$$

We choose

$$
0<\theta \leq \frac{1}{4}
$$

that is,

$$
\frac{8+16 \theta}{3} \leq 4
$$

Thus

$$
\|u\|_{L^{4}}^{(8+16 \theta) / 3} \leq C\left(\|u\|_{L^{4}}^{4}+1\right)
$$

which together with (27) gives

$$
\begin{aligned}
& \|\pi\|_{L^{4}}^{2}\|u\|_{L^{4}}^{2} \\
& \quad \leq C\left(\left\|\partial_{3} \pi\right\|_{L^{q}}^{(2(1-\theta)) /(1+2 \theta)}+\|\nabla u\|_{L^{2}}^{2}\right)\left(\|u\|_{L^{4}}^{4}+1\right) .
\end{aligned}
$$

Collecting (21), (22), and (31), we derive

$$
\begin{aligned}
& \frac{d}{d t}\left(\int_{\mathbf{R}^{3}}|u|^{4} d x+\int_{\mathbf{R}^{3}}|w|^{4} d x\right) \\
& \quad+\frac{\nu+\kappa}{2} \int_{\mathbf{R}^{3}}|| u|\nabla u|^{2} d x+\frac{\gamma}{2} \int_{\mathbf{R}^{3}}|| w|\nabla w|^{2} d x \\
& \quad \leq C\left(\left\|\partial_{3} \pi\right\|_{L^{q}}^{(2(1-\theta)) /(1+2 \theta)}+\|\nabla u\|_{L^{2}}^{2}\right)\left(\|u\|_{L^{4}}^{4}+1\right)
\end{aligned}
$$

or

$$
\begin{aligned}
\frac{d}{d t}\left(\|u\|_{L^{4}}^{4}+\|w\|_{L^{4}}^{4}+1\right) \\
\leq C\left(\left\|\partial_{3} \pi\right\|_{L^{q}}^{(2(1-\theta)) /(1+2 \theta)}+\|\nabla u\|_{L^{2}}^{2}\right) \\
\quad \times\left(\|u\|_{L^{4}}^{4}+\|w\|_{L^{4}}^{4}+1\right) .
\end{aligned}
$$

Taking Gronwall inequality into consideration yields

$$
\begin{aligned}
\text { ess } \sup _{0<t<T}\left(\|u(t)\|_{L^{4}}^{4}+\|w(t)\|_{L^{4}}^{4}\right) \\
\leq\left(\left\|u_{0}\right\|_{L^{4}}^{4}+\left\|w_{0}\right\|_{L^{4}}^{4}+1\right) \\
\quad \times \exp \left\{C \int_{0}^{T}\left(\left\|\partial_{3} \pi\right\|_{L^{q}}^{(2(1-\theta)) /(1+2 \theta)}+\|\nabla u\|_{L^{2}}^{2}\right) d \tau\right\} .
\end{aligned}
$$

By the definitions of the weak solutions, we have

$$
\int_{0}^{T}\|\nabla u(\tau)\|_{L^{2}}^{2} d \tau \leq C\left(\left\|u_{0}\right\|_{L^{2}},\left\|w_{0}\right\|_{L^{2}}\right) .
$$

Letting $p=(2(1-\theta)) /(1+2 \theta)$, we could obtain

$$
\frac{2}{p}+\frac{3}{q}=2-\frac{1-4 \theta}{4(1-\theta)} \leq 2,
$$

which exactly satisfies the growth condition (13) in Theorem 4 ; therefore, we derive the uniform bounds of $(u, w)$

$$
\text { ess } \sup _{0<t<T}\left(\|u(t)\|_{L^{4}}^{4}+\|w(t)\|_{L^{4}}^{4}\right) \leq C,
$$

together with Lemma 3; we now complete the proof of Theorem 4.

\section{Acknowledgments}

The author would like to express his sincere thanks to the editors and the referees for their valuable comments and suggestions. This work is partially supported by the NNSF of China grants nos. 11361026 and 11161022 .

\section{References}

[1] O. A. Ladyzhenskaya, The Mathematical Theory of Viscous Incompressible Fluids, Gorden Brech, New York, NY, USA, 1969.

[2] S. Popel, A. Regirer, and P. Usick, "A continuum model of blood flow," Biorheology, vol. 11, pp. 427-437, 1974.

[3] A. C. Eringen, "Theory of micropolar fluids," Journal of Applied Mathematics and Mechanics, vol. 16, pp. 1-18, 1966.

[4] B.-Q. Dong and Z.-M. Chen, "Asymptotic profiles of solutions to the $2 \mathrm{D}$ viscous incompressible micropolar fluid flows," Discrete and Continuous Dynamical Systems A, vol. 23, no. 3, pp. 765784, 2009.

[5] Z. Q. Luo, "Numerical solution of potential flow equations with predictor-corrector finite difference method," Journal of Zhejiang University Science C, vol. 13, pp. 393-402, 2012. 
[6] B.-Q. Dong and Z. Zhang, "Global regularity of the 2D micropolar fluid flows with zero angular viscosity," Journal of Differential Equations, vol. 249, no. 1, pp. 200-213, 2010.

[7] Z. Q. Luo and Z. M. Chen, "Numerical simulation of standing wave with $3 \mathrm{D}$ predictor-corrector finite difference method for potential flow equations," Applied Mathematics and Mechanics, vol. 35, pp. 931-944, 2013.

[8] G. P. Galdi and S. Rionero, "A note on the existence and uniqueness of solutions of the micropolar fluid equations," International Journal of Engineering Science, vol. 15, no. 2, pp. 105$108,1977$.

[9] G. Łukaszewicz, Micropolar Fluids. Theory and Applications, Modeling and Simulation in Science, Engineering and Technology, Birkhäuser, Boston, Mass, USA, 1999.

[10] Z.-q. Luo and Z.-m. Chen, "Sloshing simulation of standing wave with time-independent finite difference method for Euler equations," Applied Mathematics and Mechanics, vol. 32, no. 11, pp. 1475-1488, 2011.

[11] B.-Q. Dong and Z.-M. Chen, "Regularity criteria of weak solutions to the three-dimensional micropolar flows," Journal of Mathematical Physics, vol. 50, no. 10, Article ID 103525, 2009.

[12] B. Yuan, "On regularity criteria for weak solutions to the micropolar fluid equations in Lorentz space," Proceedings of the American Mathematical Society, vol. 138, no. 6, pp. 2025-2036, 2010.

[13] B.-Q. Dong and W. Zhang, "On the regularity criterion for three-dimensional micropolar fluid flows in Besov spaces," Nonlinear Analysis: Theory, Methods \& Applications, vol. 73, no. 7, pp. 2334-2341, 2010.

[14] S. Gala, "Regularity criteria for the 3D magneto-micropolar fluid equations in the Morrey-Campanato space," Nonlinear Differential Equations and Applications, vol. 17, no. 2, pp. 181194, 2010.

[15] Z. Zhang, Z.-a. Yao, and X. Wang, "A regularity criterion for the 3D magneto-micropolar fluid equations in Triebel-Lizorkin spaces," Nonlinear Analysis: Theory, Methods \& Applications, vol. 74, no. 6, pp. 2220-2225, 2011.

[16] B.-Q. Dong, Y. Jia, and Z.-M. Chen, "Pressure regularity criteria of the three-dimensional micropolar fluid flows," Mathematical Methods in the Applied Sciences, vol. 34, no. 5, pp. 595-606, 2011.

[17] Y. Jia, W. Zhang, and B.-Q. Dong, "Remarks on the regularity criterion of the 3D micropolar fluid flows in terms of the pressure," Applied Mathematics Letters, vol. 24, no. 2, pp. 199203, 2011.

[18] F. Guo, "Remarks on the pressure regularity criterion of the micropolar fluid equations in multiplier spaces," Abstract and Applied Analysis, Article ID 618084, 10 pages, 2012.

[19] F. Xu, X. Xu, and J. Yuan, "Logarithmically improved regularity criteria for the micropolar fluid equations," Applicationes Mathematicae, vol. 39, no. 3, pp. 315-328, 2012.

[20] Y. Jia, J. Zhang, and B.-Q. Dong, "Logarithmical regularity criteria of the three-dimensional micropolar fluid equations in terms of the pressure," Abstract and Applied Analysis, vol. 2012, Article ID 395420, 13 pages, 2012.

[21] C. Cao and E. S. Titi, "Global regularity criterion for the 3D Navier-Stokes equations involving one entry of the velocity gradient tensor," Archive for Rational Mechanics and Analysis, vol. 202, no. 3, pp. 919-932, 2011.

[22] Q. Chen and Z. Zhang, "Regularity criterion via the pressure on weak solutions to the 3D Navier-Stokes equations," Proceedings of the American Mathematical Society, vol. 135, no. 6, pp. 18291837, 2007.
[23] J. Fan, S. Jiang, and G. Ni, "On regularity criteria for the ndimensional Navier-Stokes equations in terms of the pressure," Journal of Differential Equations, vol. 244, no. 11, pp. 2963-2979, 2008.

[24] Y. Zhou, "On regularity criteria in terms of pressure for the Navier-Stokes equations in $R^{3}$," Proceedings of the American Mathematical Society, vol. 134, no. 1, pp. 149-156, 2006.

[25] Q. Chen, C. Miao, and Z. Zhang, "On the regularity criterion of weak solution for the $3 \mathrm{D}$ viscous magneto-hydrodynamics equations," Communications in Mathematical Physics, vol. 284, no. 3, pp. 919-930, 2008.

[26] C. He and Z. Xin, "On the regularity of weak solutions to the magnetohydrodynamic equations," Journal of Differential Equations, vol. 213, no. 2, pp. 235-254, 2005.

[27] X. Jia and Y. Zhou, "A new regularity criterion for the 3D incompressible MHD equations in terms of one component of the gradient of pressure," Journal of Mathematical Analysis and Applications, vol. 396, no. 1, pp. 345-350, 2012.

[28] Y. Zhou, "Regularity criteria for the 3D MHD equations in terms of the pressure," International Journal of Non-Linear Mechanics, vol. 41, no. 10, pp. 1174-1180, 2006.

[29] B.-Q. Dong and Z.-M. Chen, "A remark on regularity criterion for the dissipative quasi-geostrophic equations," Journal of Mathematical Analysis and Applications, vol. 329, no. 2, pp. 12121217, 2007.

[30] H. Dong and N. Pavlović, "Regularity criteria for the dissipative quasi-geostrophic equations in Hölder spaces," Communications in Mathematical Physics, vol. 290, no. 3, pp. 801-812, 2009.

[31] C. Cao and J. Wu, "Two regularity criteria for the 3D MHD equations," Journal of Differential Equations, vol. 248, no. 9, pp. 2263-2274, 2010. 


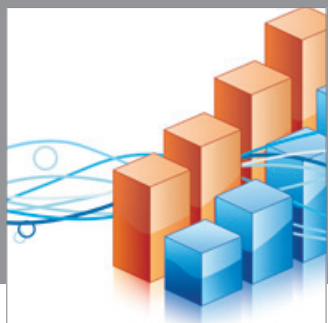

Advances in

Operations Research

mansans

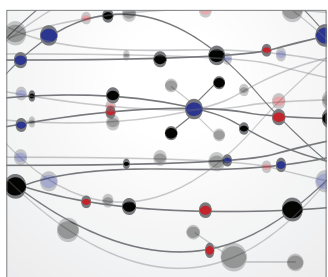

The Scientific World Journal
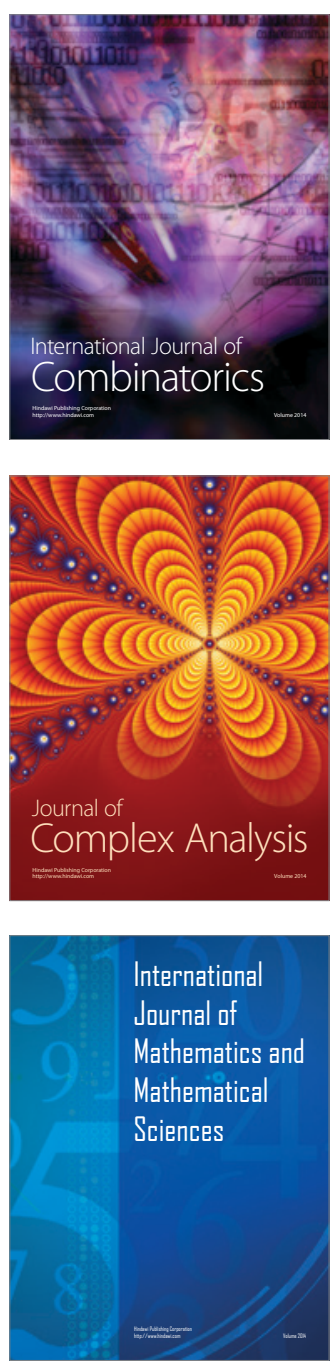
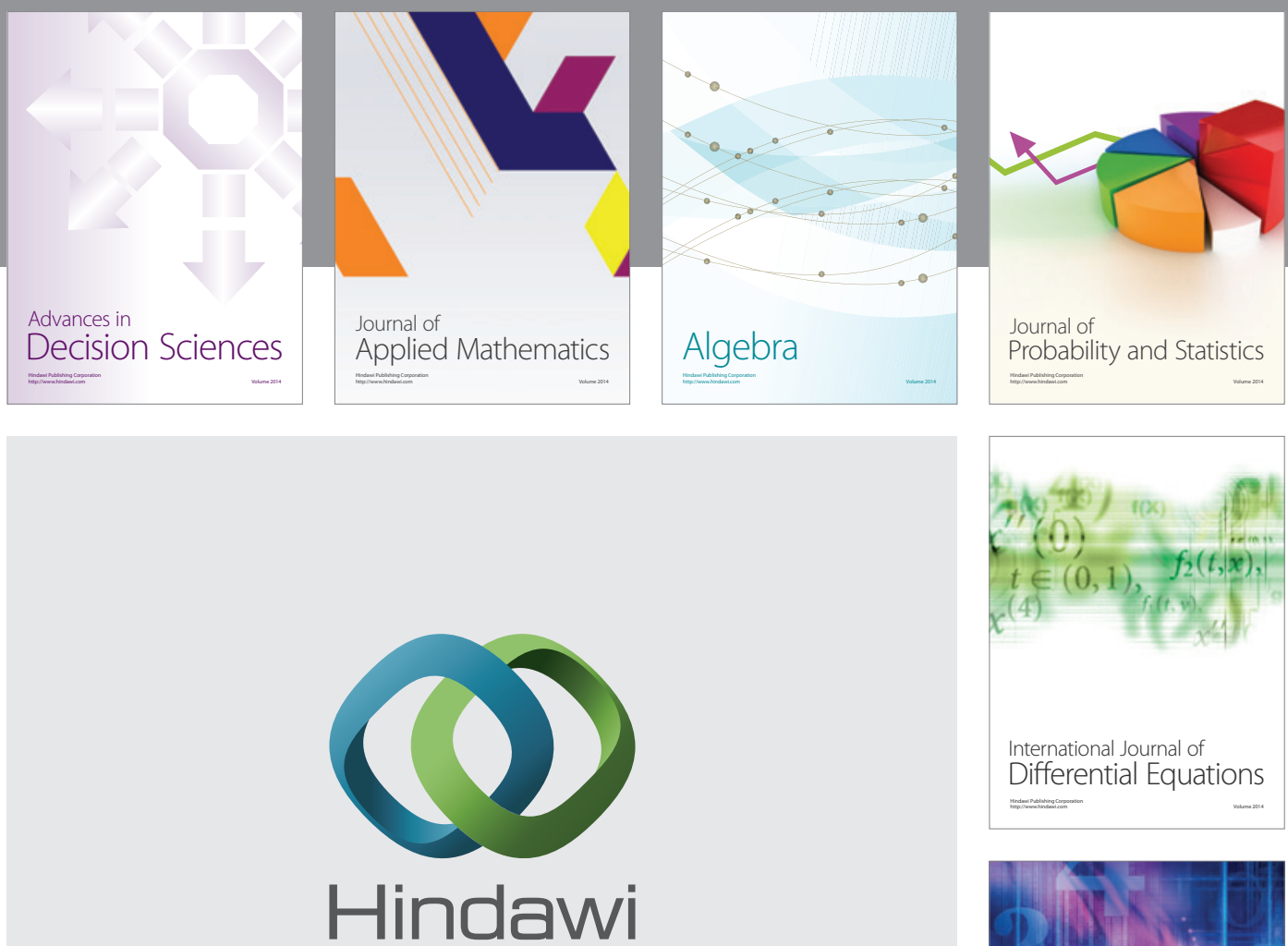

Submit your manuscripts at http://www.hindawi.com
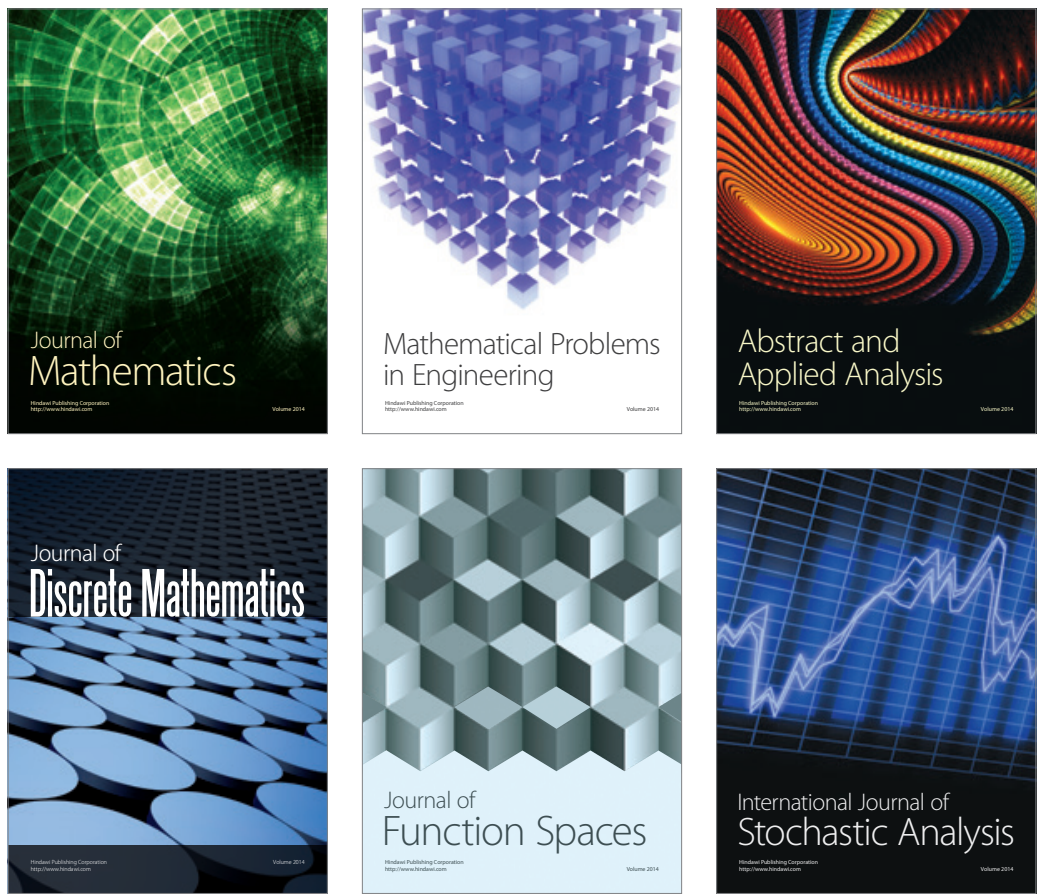

Journal of

Function Spaces

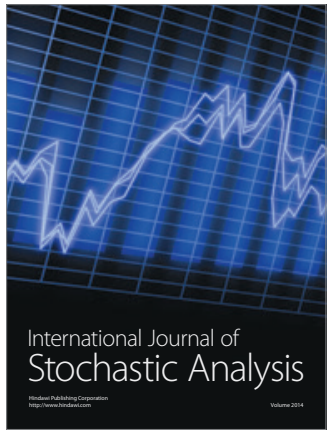

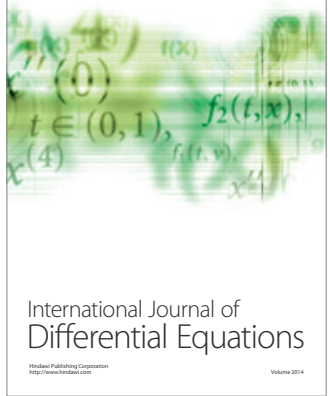
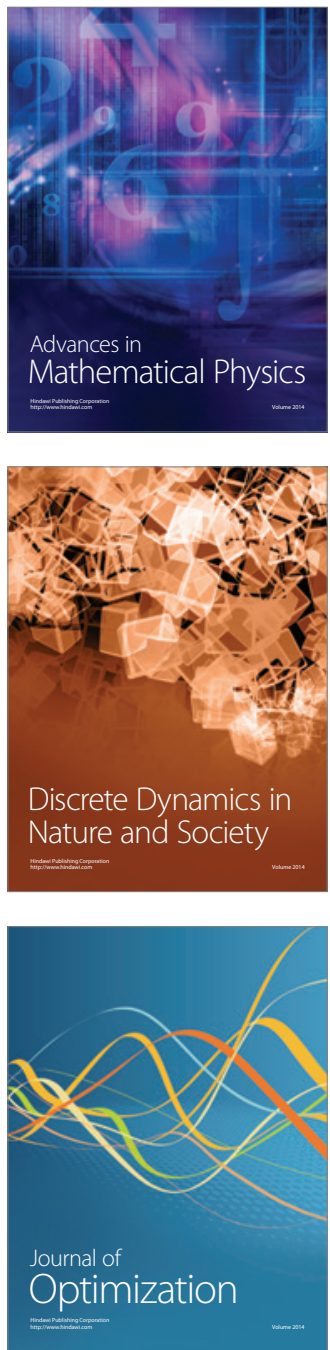